\title{
Photoproduction of $f_{0}(980)$ and $f_{0}(1500)$ resonances off a proton target
}

\author{
Hanyang Xing, ${ }^{1,2}$ Chun-Sheng An, ${ }^{1} \mathrm{Ju}-\mathrm{Jun} \mathrm{Xie},{ }^{2}$ and Gang $\mathrm{Li}^{3, *}$ \\ ${ }^{1}$ School of Physical Science and Technology, Southwest University, Chongqing 400715, China \\ ${ }^{2}$ Institute of Modern Physics, Chinese Academy of Sciences, Lanzhou 730000, China \\ ${ }^{3}$ School of Physics and Engineering, Qufu Normal University, Qufu 273165, China
}

(Received 8 August 2018; published 12 November 2018)

\begin{abstract}
We study the $\gamma p \rightarrow p f_{0}\left[f_{0}(980)\right.$ and $\left.f_{0}(1500)\right]$ reaction close to threshold within an effective Lagrangian approach. The production process is described by an $s$-channel nucleon pole or $t$-channel $\rho$ and $\omega$ exchange. The $K^{0} \bar{K}^{0}$ invariant mass distributions of the $\gamma p \rightarrow f_{0}(980) p \rightarrow K^{0} \bar{K}^{0}$ and $\gamma p \rightarrow$ $f_{0}(1500) p \rightarrow K^{0} \bar{K}^{0} p$ reactions are investigated, where the two kaons have been separated in the $S$ wave decaying from $f_{0}(980)$ and $f_{0}(1500)$. It is shown that the $s$-channel process is favored for the production of $f_{0}(980)$, while for the $f_{0}(1500)$ production, the experimental measurements can be described quite well by the $t$-channel process. It is expected that the theoretical results can be tested by further experiments at CLAS.
\end{abstract}

DOI: 10.1103/PhysRevD.98.094007

\section{INTRODUCTION}

The study of the structure of low-lying scalar mesons is a topic of high interest in hadronic physics and is attracting much attention $[1,2]$. For the scalar meson $f_{0}(980)$, it is now widely accepted that the simplest picture, where it is described as an orbital excitation of quark-antiquark pairs, is not compatible with the experimental observations on its decay modes. Thus, the $f_{0}(980)$ is thought to be a molecule state formed from the interaction of pseudoscalar mesons [3-10], and it couples strongly to the $K \bar{K}$ channel [11], which is its dominant component. The $f_{0}(1500)$, on the other hand, with a mass of $1504 \pm 6 \mathrm{MeV}$ and a width of $109 \pm 7 \mathrm{MeV}$ [12], is a candidate for having significant glueball content $[13,14]$. Photoproduction of these scalar resonances provides a unique place to probe their nature.

On the experimental side, photoproduction of the $f_{0}(980)$ meson on protons was measured by the CLAS Collaboration in Refs. $[15,16]$ at the photon energy region of $E_{\gamma}=3.0-3.8 \mathrm{GeV}$, where $f_{0}(980)$ was detected via its decay in the $\pi^{+} \pi^{-}$channel by performing a partial wave analysis of the reaction $\gamma p \rightarrow p \pi^{+} \pi^{-}$. However, the production rate of $f_{0}(980)$ is much smaller than the one for the $\rho$ meson. Very recently, a partial wave analysis was performed for the $\gamma p \rightarrow p K^{+} K^{-}$reaction by the CLAS Collaboration [17], where the production amplitudes have

*gli@qfnu.edu.cn

Published by the American Physical Society under the terms of the Creative Commons Attribution 4.0 International license. Further distribution of this work must maintain attribution to the author(s) and the published article's title, journal citation, and DOI. Funded by SCOAP ${ }^{3}$. been parametrized using a Regge-theory inspired model. There were also pioneering measurements $[18,19]$ for the photoproduction of $K^{+} K^{-}$pairs. After that, there were several theoretical calculations about the scalar meson production in the process of $\gamma p$ scattering. A combined analysis of $\pi \pi$ and $K \bar{K}$ photoproduction in $S$ wave is conducted in Ref. [20], while the $f_{0}(980)$ and $a_{0}(980)$ photoproduction for photon energies close to the $K \bar{K}$ production threshold was studied in Ref. [21] using tools of chiral unitary approach. In Ref. [22], within a model based on the Regge approach, a theoretical analysis of the data on photoproduction of the $f_{0}(980)$ was done, where it was shown that the radiative decay rate for $f_{0}(980) \rightarrow \gamma V$ is important in the theoretical predictions. In Ref. [23], the $\gamma p \rightarrow a_{0}(980) p$ and $\gamma p \rightarrow f_{0}(980) p$ reactions were investigated with the main aim of studying the possibility of observing $a_{0}(980)-f_{0}(980)$ mixing in these processes. In Ref. [24], the $a_{0}(980)$ and $f_{0}(980)$ photoproduction was investigated by considering the Regge-cut effects which were fixed from $\pi^{0}$ photoproduction. With the Regge theory, the $f_{0}(1500)$ photoproduction was also studied in Ref. [25] at $E_{\gamma}=9 \mathrm{GeV}$.

Recently, the reaction $\gamma p \rightarrow p X \rightarrow p K_{S}^{0} K_{S}^{0}$ was investigated by the CLAS Collaboration [26] with photon energies of 2.7-5.1 GeV, where it was found that the angular distributions of the data suggest that most of the $K_{S}^{0} K_{S}^{0}$ decay is from scalar mesons in $S$ wave. In particular, a clear peak is seen at $1500 \mathrm{MeV}$ in the invariant mass spectra of $K_{S}^{0} K_{S}^{0}$, and the mass and width of this peak is consistent with that of the scalar meson $f_{0}(1500)$, while the enhancement close to $K_{S}^{0} K_{S}^{0}$ threshold is due to the $f_{0}(980)$ decay. In addition, there is no clear signals for contributions from the baryon resonances. 
In the present work, based on the new measurements of CLAS collaboration [26], we reanalyze the $\gamma p \rightarrow$ $f_{0}(980) p \rightarrow K^{0} \bar{K}^{0} p \quad$ and $\quad \gamma p \rightarrow f_{0}(1500) p \rightarrow K^{0} \bar{K}^{0} p$ reactions ${ }^{1}$ within an effective Lagrangian method near threshold. As in Refs. [22,23], we consider the contributions from $t$-channel $\rho^{0}$ and $\omega$ exchange. Since the couplings of $f_{0}$ to the $V \gamma$ channel is scarce [12], we take these results obtained in Refs. [27,28], where meson loops were considered, and the $f_{0}(980)$ was taken as a dynamically generated state. On the other hand, a possible $s$-channel proton pole process, which was not included in all these above theoretical calculations, is also investigated in this work. It is shown that the new measurements of Ref. [26] may indicate the dominant $s$-channel contribution for the $f_{0}(980)$ photoproduction. In this respect, we show in this work how the CLAS measurements could be used to determine the reaction mechanisms of the photoproduction of these scalar mesons.

In the next section, we will give the formalism and ingredients in this work, then numerical results and discussions are given in Sec. III. A short summary is given in the last section.

\section{FORMALISM AND INGREDIENTS}

The effective Lagrangian method is widely used to calculate cross sections for different reactions in the resonance production region. In this section, we introduce theoretical formalism and ingredients to calculate the scalar mesons photoproduction off protons within the effective Lagrangian method.

\section{A. Interaction Lagrangian densities and scattering amplitudes}

We first consider the basic $t$-channel tree-level diagram for the $\gamma p \rightarrow p f_{0}\left[f_{0} \equiv f_{0}(980)\right.$ or $\left.f_{0}(1500)\right]$ reaction as shown in Fig. 1. This includes the contributions from $\rho^{0}$ and $\omega$ meson exchange terms.

Following Ref. [28], we can write down the amplitude for the $f_{0} \rightarrow V \gamma$ deacy as

$$
T=-\frac{g_{f_{0} V \gamma}}{m_{f_{0}}}\left(k \cdot p_{1} g^{\mu \nu}-k^{\mu} p_{1}^{\nu}\right) \varepsilon_{V \mu}(k) \varepsilon_{\nu}\left(p_{1}\right),
$$

from where, we can obtain the partial decay width of the $f_{0}$ meson into a vector meson and a photon,

$$
\begin{aligned}
\Gamma_{f_{0} \rightarrow V \gamma} & =\frac{|\vec{k}|}{8 \pi M_{f_{0}}^{2}} \sum \sum|T|^{2} \\
& =\frac{g_{f_{0} V \gamma}^{2}}{32 \pi} \frac{\left(M_{f_{0}}^{2}-m_{V}^{2}\right)^{3}}{M_{f_{0}}^{5}} .
\end{aligned}
$$

\footnotetext{
${ }^{1}$ We take $\left|K^{0}\right\rangle=\frac{1}{\sqrt{2}}\left(\left|K_{S}^{0}\right\rangle+\left|K_{L}^{0}\right\rangle\right)$ and $\left|\bar{K}^{0}\right\rangle=\frac{1}{\sqrt{2}}\left(\left|K_{S}^{0}\right\rangle-\left|K_{L}^{0}\right\rangle\right)$, where we ignore the $C P$ violation.
}

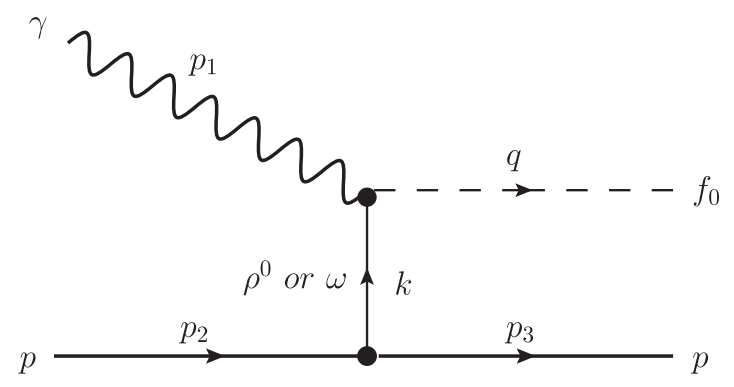

FIG. 1. Schematic diagram of reaction mechanism for $\gamma p \rightarrow$ $f_{0} p$ reaction with $t$-channel $\rho^{0}$ and $\omega$ exchange. The definition of the kinematical variables $\left(p_{1}, p_{2}, p_{3}, q\right)$ used in the present calculation are also shown.

With masses $\quad\left(M_{f_{0}(980)}=990 \mathrm{MeV}, \quad M_{f_{0}(1500)}=\right.$ $1504 \mathrm{MeV}$, and $m_{\rho}=m_{\omega}=m_{V}=780 \mathrm{MeV}$ ), and the partial decay widths of the scalar $f_{0}(980)$ and $f_{0}(1500)$ mesons radiative decay into a vector meson and a photon as obtained in Refs. [27,28], we obtain these coupling constants as list in Table I.

To compute the scattering amplitudes of the diagrams shown in Fig. 1, we need also the effective interactions for the $\rho N N$ and $\omega N N$ vertices. We take the interaction Lagrangian densities as used in Refs. [29,30]:

$$
\begin{gathered}
\mathcal{L}_{\rho N N}=-g_{\rho N N} \bar{N}\left(\gamma^{\mu}-\frac{\kappa_{\rho}}{2 m_{N}} \sigma^{\mu \nu} \partial_{\nu}\right) \vec{\tau} \cdot \vec{\rho}_{\mu} N, \\
\mathcal{L}_{\omega N N}=-g_{\omega N N} \bar{N}\left(\gamma^{\mu}-\frac{\kappa_{\omega}}{2 m_{N}} \sigma^{\mu \nu} \partial_{\nu}\right) \omega_{\mu} N .
\end{gathered}
$$

We use the coupling constants $g_{\rho N N}=3.36, \kappa_{\rho}=6.1$, $g_{\omega N N}=15.85$ and $\kappa_{\omega}=0$ of Refs. [31,32]. Then we can write the $\rho N N$ and $\omega N N$ vertices as,

$$
\begin{aligned}
&-\mathrm{i} t_{\rho N N}= \mathrm{i} g_{\rho N N}\left(\gamma^{\mu}+\mathrm{i} \frac{\kappa_{\rho}}{2 m_{N}} \sigma^{\mu \nu} q_{\nu}\right) \varepsilon_{\mu}(\rho), \\
&-\mathrm{i} t_{\omega N N}=\mathrm{i} g_{\omega N N} \gamma^{\mu} \varepsilon_{\mu}(\omega) .
\end{aligned}
$$

\section{B. $\gamma p \rightarrow p f_{0}$ scattering amplitudes}

With ingredients given above, we can easily obtain the $t$-channel $\gamma p \rightarrow f_{0} p$ reaction invariant scattering amplitude:

TABLE I. Values of the coupling constants required for the estimation of the $\gamma p \rightarrow p f_{0}$ reaction.

\begin{tabular}{lcc}
\hline \hline Decay channels & Partial decay width $\Gamma_{f_{0} \rightarrow V \gamma}(\mathrm{keV})$ & $g_{f_{0} V_{\gamma}}$ \\
\hline$f_{0}(980) \rightarrow \rho \gamma$ & $7.3 \pm 1.8$ & 0.12 \\
$f_{0}(980) \rightarrow \omega \gamma$ & $6.6 \pm 1.8$ & 0.11 \\
$f_{0}(1500) \rightarrow \rho \gamma$ & $77 \pm 8$ & 0.11 \\
$f_{0}(1500) \rightarrow \omega \gamma$ & $79 \pm 8$ & 0.12 \\
\hline \hline
\end{tabular}




$$
\begin{aligned}
\mathcal{M}_{V}= & -\bar{u}\left(p_{3}\right) \frac{g_{f_{0} V \gamma}}{m_{f_{0}}} g_{V N N}\left(k \cdot p_{1} g^{\nu \sigma}-k^{\nu} p_{1}^{\sigma}\right) G_{\mu \nu} \\
& \times\left[\gamma_{\mu}+\frac{\kappa_{V}}{2 m_{p}}\left(k_{\mu}-\not k \gamma_{\mu}\right)\right] F_{1} u\left(p_{2}, s_{p}\right) \varepsilon_{\sigma}\left(p_{1}\right),
\end{aligned}
$$

where $G_{\mu \nu}$ is the Feynman propagator of $\rho$ or $\omega$ meson which has the following form:

$$
G_{\mu \nu}=-\mathrm{i} \frac{g_{\mu \nu}-k_{\mu} k_{\nu} / m_{V}^{2}}{k^{2}-m_{V}^{2}}
$$

Since hadrons are not pointlike particles, the form factor of hadrons need to be taken into account [32,33]:

$$
F_{1}=\left(\frac{\Lambda_{c}^{2}-m_{V}^{2}}{\Lambda_{c}^{2}-t}\right)^{2}
$$

with $t=k^{2}$ and $\Lambda_{c}$ a free cutoff parameter.

\section{Differential cross section}

The differential cross section for the $\gamma p \rightarrow p f_{0}$ reaction by the exchanged $\rho^{0}$ and $\omega$ mesons can be expressed as

$$
\frac{d \sigma}{d t}=\frac{1}{16 \pi s} \frac{m_{p}^{2}}{\left|\vec{p}_{1}\right|^{2}}\left(\frac{1}{4} \sum|\mathcal{M}|^{2}\right)
$$

where $s$ is the invariant mass square of the $\gamma p$ system, and $\vec{p}_{1}$ denotes the photon three momentum in the center of mass (c.m.) frame. The total invariant scattering amplitude $\mathcal{M}$ is given by

$$
\begin{aligned}
\sum|\mathcal{M}|^{2} & =\sum\left|\mathcal{M}_{\rho}+\mathcal{M}_{\omega}\right|^{2} \\
& =\frac{1}{4 m_{p}^{2}} \sum_{V_{1}, V_{2}=\rho, \omega} \operatorname{Tr}\left[\left(\not p_{3}+m_{3}\right) \Gamma_{V_{1}}^{\mu}\left(\not \not_{2}+m_{2}\right) \Gamma_{V_{2}}^{\nu} g_{\mu \nu}\right]
\end{aligned}
$$

with

$$
\begin{aligned}
\Gamma_{V}^{\mu}= & \frac{g_{V N N} g_{f_{0} V \gamma}}{\left(t-m_{V}^{2}\right) M_{f_{0}}}\left[\left(1+\kappa_{V}\right) p_{1} \cdot k \gamma^{\mu}-\left(1+\kappa_{V}\right) \not p_{1} k^{\mu}\right. \\
& \left.+\frac{\kappa_{V}}{2 M_{N}} p_{1} \cdot k\left(p_{2}^{\mu}+p_{3}^{\mu}\right)+\frac{\kappa_{V}}{2 M_{N}} p_{1} \cdot\left(p_{2}+p_{3}\right) k^{\mu}\right] .
\end{aligned}
$$

On the other hand, we can generalize the two body process as in Eq. (10) by considering the situation which allows the $f_{0}$ to decay into a $K^{0}$ and a $\bar{K}^{0}$ as shown in Fig. 2. By working out the three-body phase space of the $\gamma p \rightarrow f_{0} p \rightarrow p K^{0} \bar{K}^{0}$ reaction, we find

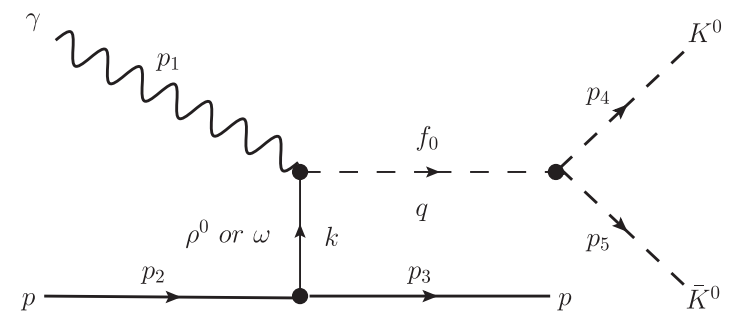

FIG. 2. Feynman diagram for $t$-channel $\gamma p \rightarrow f_{0} p \rightarrow K^{0} \bar{K}^{0} p$ reaction.

$\frac{d^{2} \sigma}{d M_{\mathrm{inv}} d t}=\frac{m_{p}^{2}}{32 \pi^{2}} \frac{M_{\mathrm{inv}}^{2}}{s\left|\vec{p}_{1}\right|^{2}}|\mathcal{M}|^{2} \frac{\Gamma_{f_{0} \rightarrow K^{0} \bar{K}^{0}}}{\left(M_{\mathrm{inv}}^{2}-M_{f_{0}}^{2}\right)^{2}+M_{f_{0}}^{2} \Gamma_{f_{0}}^{2}}$,

where $\Gamma_{f_{0}}$ is the total decay width and we take $\Gamma_{f_{0}}=100 \mathrm{MeV}^{2}$ and $109 \mathrm{MeV}$ for $f_{0}(980)$ and $f_{0}(1500)$, respectively. $M_{\text {inv }}$ represents the invariant mass of $K^{0} \bar{K}^{0}$. For $f_{0}(1500), \Gamma_{f_{0} \rightarrow K^{0} \bar{K}^{0}}$ is given by

$$
\Gamma_{f_{0} \rightarrow K^{0} \bar{K}^{0}}=\Gamma_{K^{0} \bar{K}^{0}}^{\mathrm{on}} \sqrt{\frac{M_{\mathrm{inv}}^{2}-4 m_{K^{0}}^{2}}{M_{f_{0}}^{2}-4 m_{K^{0}}^{2}}} \frac{M_{f_{0}}^{2}}{M_{\mathrm{inv}}^{2}},
$$

with $\Gamma_{K^{0} \bar{K}^{0}}^{\text {on }}=4.7 \mathrm{MeV}$. While for the case of $f_{0}(980)$, we take

$$
\Gamma_{f_{0} \rightarrow K^{0} \bar{K}^{0}}=\frac{g_{f_{0} K \bar{K}}^{2}}{16 \pi} \frac{\sqrt{M_{\mathrm{inv}}^{2}-4 m_{K^{0}}^{2}}}{2 M_{\mathrm{inv}}^{2}},
$$

with $g_{f_{0}(980) K \bar{K}}=3860 \mathrm{MeV}$ as in Refs. [28,34,35].

\section{NUMERICAL RESULTS AND DISCUSSION}

In this section, we will show the numerical results for the $\gamma p \rightarrow p f_{0}$ reaction. We first show the theoretical results for the case of $f_{0}(1500)$ photoproduction. There is also another peak around 1.28 GeV (see Ref. [26] for more details). However, the width of the observed peak is much narrower than the average PDG listed width of the $f_{2}(1270)$ or $f_{0}(1370)$, which have a wide width [12]: $\Gamma_{f_{2}(1270)}=$ $185.9_{-2.1}^{+2.9} \mathrm{MeV}$ and $\Gamma_{f_{0}(1370)}=200 \sim 500 \mathrm{MeV}$. Thus, it is not clear if this bump indicates a resonance of something else, and we will not take it into account in this work.

\section{A. Invariant mass distributions for the $\gamma p \rightarrow p f_{0}(1500) \rightarrow p K^{0} \bar{K}^{0}$ reaction}

We compare our theoretical calculations for the invariant $K^{0} \bar{K}^{0}$ mass distributions as a function of $M_{\text {inv }}$ with the recent CLAS data of Ref. [26]. The theoretical $d \sigma / d M_{\text {inv }}$ is calculated by

\footnotetext{
${ }^{2}$ We take a relative large value for the total decay width of the $f_{0}(980)$, which is favored by the new CLAS measurements [26].
} 

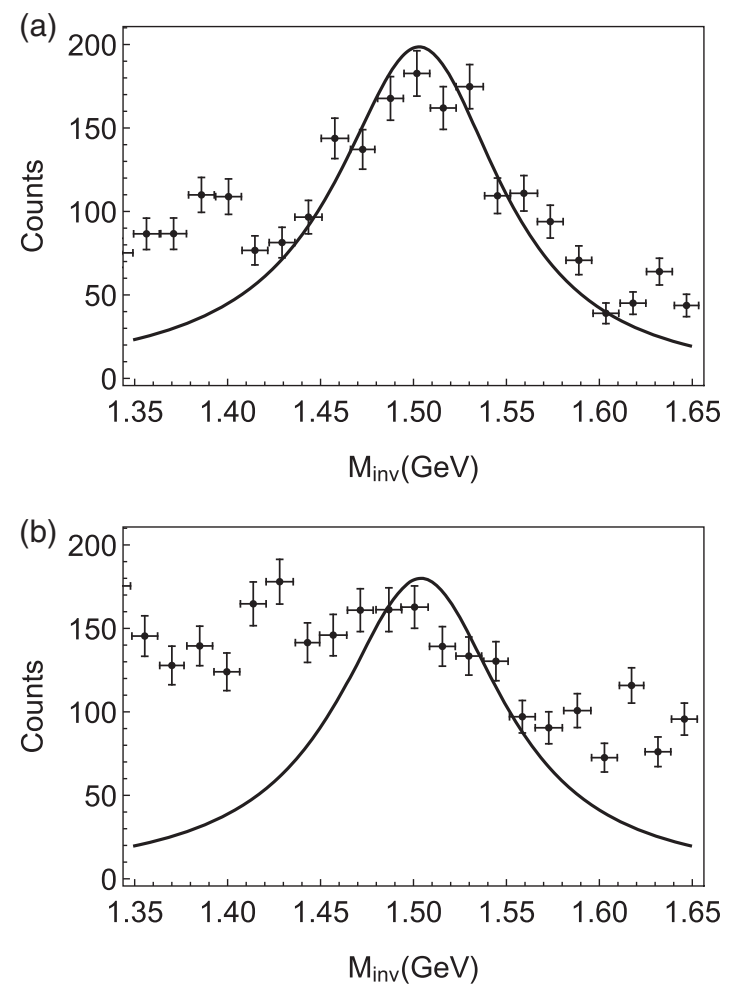

FIG. 3. Invariant mass distribution of $K^{0} \bar{K}^{0}$ of $\gamma p \rightarrow$ $p f_{0}(1500) \rightarrow p K^{0} \bar{K}^{0}$ reaction for (a) $|t|<1.0 \mathrm{GeV}^{2}$ and (b) $|t|>1.0 \mathrm{GeV}^{2}$.

$$
\frac{d \sigma}{d M_{\mathrm{inv}}}=\frac{\int_{E_{\gamma}^{\min }}^{E_{\max }} d E_{\gamma} \int d t \frac{\mathrm{d}^{2} \sigma}{d M_{\mathrm{inv}} d t}}{\int_{E_{\gamma}^{\min }}^{E_{\text {max }}^{\max }} d E_{\gamma}}
$$

with $E_{\gamma}^{\max }=5.1 \mathrm{GeV}$ and $E_{\gamma}^{\min }=2.7 \mathrm{GeV}$, which are the photon energy regions of Ref. [26].

In Fig. 3, we show the theoretical results, $c_{1} d \sigma / d M_{\text {inv }}$, for the $K^{0} \bar{K}^{0}$ invariant mass distributions for the $\gamma p \rightarrow$ $p f_{0}(1500) \rightarrow p K^{0} \bar{K}^{0}$ reaction, compared with the experimental measurements of Ref. [26], where $c_{1}=2.2$ and $\Lambda_{c}=1.7 \mathrm{GeV}$ have been adjusted to the strength of the experimental data reported by the CLAS Collaboration [26] at its peak around $M_{\text {inv }}=1500 \mathrm{MeV}$. One can see that we can describe quite well the experimental measurements for the $\gamma p \rightarrow p f_{0}(1500) \rightarrow p K^{0} \bar{K}^{0}$ reaction by considering the $t$-channel $\rho^{0}$ and $\omega$ exchange, especially for the case of $|t|<1 \mathrm{GeV}^{2}$. This may indicate that the $t$-channel process is dominant for the photoproduction of the $f_{0}(1500)$ resonance.

For a meson with a large glueball admixture, it is expected that its photon coupling in the $t$ channel is suppressed, since its wave function contains a glueball component. Based on our calculations, the $f_{0}(1500)$ resonance is mostly produced from the $t$-channel vector meson exchange process; thus, it is speculated that the glueball content of $f_{0}(1500)$ is not large. However, we
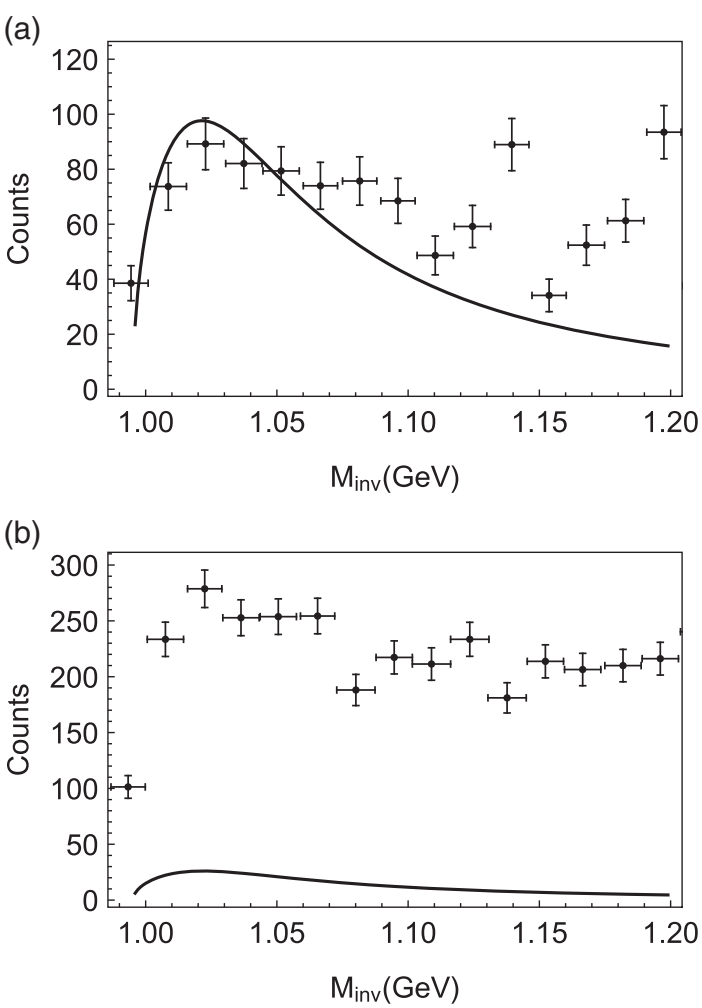

FIG. 4. Invariant mass distribution of $K^{0} \bar{K}^{0}$ of $\gamma p \rightarrow$ $p f_{0}(980) \rightarrow p K^{0} \bar{K}^{0}$ reaction for (a) $|t|<1.0 \mathrm{GeV}^{2}$ and (b) $|t|>1.0 \mathrm{GeV}^{2}$. The theoretical results are obtained by considering only the $t$ channel $\rho^{0}$ and $\omega$ exchange.

would like to mention that there is also evidence that the $f_{0}(1500)$ is a mainly glueball state [36-40].

\section{B. Invariant mass distributions for the $\gamma p \rightarrow p f_{0}(980) \rightarrow p K^{0} \bar{K}^{0}$ reaction}

We first present the theoretical results for the $\gamma p \rightarrow$ $p f_{0}(980) \rightarrow p K^{0} \bar{K}^{0}$ reaction by including the $t$-channel $\rho^{0}$ and $\omega$ exchange. The numerical results of the $K^{0} \bar{K}^{0}$ invariant mass distributions obtained with $c_{1}=0.9$ and $\Lambda_{c}=1.07 \mathrm{GeV}$ are shown in Fig. 4 . The peak of the $K^{0} \bar{K}^{0}$ invariant mass distributions is around $1020 \mathrm{MeV}$, very close to the mass threshold ( $995 \mathrm{MeV}$ ) of $K^{0} \bar{K}^{0}$. One can see that the model cannot describe simultaneously the experimental data for both $|t|<1 \mathrm{GeV}^{2}$ and $|t|>1 \mathrm{GeV}^{2}$. At $M_{\text {inv }}=$ $1020 \mathrm{MeV}$ and $E_{\gamma}=3.9 \mathrm{GeV}$, the values of $t$ are $-5.36 \mathrm{GeV}^{2}<t<-0.02 \mathrm{GeV}^{2},{ }^{3}$ where we find that the phase space for $|t|>1 \mathrm{GeV}^{2}$ is more than 4 times larger than the case of $|t|<1 \mathrm{GeV}^{2}$. However, the $t$-channel form factor $F_{1}=\left(\frac{\Lambda_{c}^{2}-m_{V}^{2}}{\Lambda_{c}^{2}-t}\right)^{2}$ with $\Lambda_{c} \sim 1.07 \mathrm{GeV}$ will contribute a suppression with a factor of about 14 for the case of $|t|>$ $1 \mathrm{GeV}^{2}$ compared to that of $|t|<1 \mathrm{GeV}^{2}$. Hence, it is

\footnotetext{
${ }^{3}$ The values of $t$ for the production of $f_{0}(1500)$ is $-3.89 \mathrm{GeV}^{2}<t<-0.14 \mathrm{GeV}^{2}$.
} 


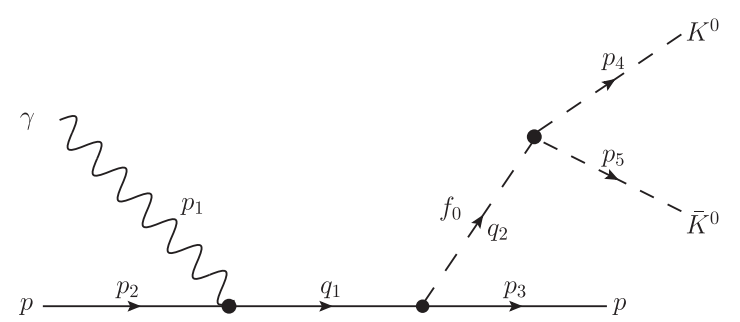

FIG. 5. Feynman diagram for the $s$-channel $\gamma p \rightarrow f_{0}(980) p \rightarrow$ $K^{0} \bar{K}^{0} p$ reaction.

expected that, with the values of $c_{1}=0.9$ and $\Lambda_{c}=1.07 \mathrm{GeV}$, the results for $|t|>1 \mathrm{GeV}^{2}$ should be much smaller than the ones for $|t|<1 \mathrm{GeV}^{2}$. But, the experimental data of Ref. [26] tell us that the values for $|t|>1 \mathrm{GeV}^{2}$ are even larger than those for $|t|<1 \mathrm{GeV}^{2}$. This may indicate that the $t$-channel exchange mechanism is not enough to explain the experimental measurements of the CLAS Collaboration [26].

We have also performed calculations for the $\gamma p \rightarrow$ $p f_{0}(980) \rightarrow p K^{0} \bar{K}^{0}$ reaction with different values of $c_{1}$ and $\Lambda_{c}$. It turns out that we can also reproduce the experimental measurements with $c_{1}=0.012$ and a large $\Lambda_{c}=5 \mathrm{GeV}$. Thus, the inclusion of another reaction mechanism is needed to achieve a good description of the new CLAS experimental measurements.

Next, we study another kind of reaction mechanism for the $\gamma p \rightarrow p f_{0}(980) \rightarrow p K^{0} \bar{K}^{0}$ reaction, which is depicted in Fig. 5, where we have considered the contribution from the $s$-channel nucleon pole term. To compute the contribution of this term, the interaction Lagrangian densities for $\gamma p p$ and $f_{0}(980) p p$ vertexes are needed. We take them as used in Refs. [23,41],

$$
\begin{gathered}
\mathcal{L}_{\gamma p p}=-e \bar{p}\left[\not A-\frac{\kappa_{p}}{2 m_{N}} \sigma^{\mu \nu}\left(\partial_{\nu} A_{\mu}\right)\right] p, \\
\mathcal{L}_{f_{0}(980) p p}=g_{f_{0} p p} \bar{p} p f_{0},
\end{gathered}
$$

where $\kappa_{p}=1.5$.

Then one can easily write down the corresponding amplitude for the $s$-channel nucleon pole term as

$$
\begin{aligned}
\mathcal{M}_{s}= & g_{f_{0} p p} F_{2} \bar{u}\left(p_{3}\right) \frac{\not q_{1}+m_{p}}{q_{1}^{2}-m_{p}^{2}} \\
& \times\left(\gamma^{\mu}-\Gamma_{c}^{\mu}-\frac{\kappa_{p}}{2 m_{p}} \gamma^{\mu} \not p_{1}\right) u\left(p_{2}\right) \varepsilon_{\mu}\left(p_{1}\right),
\end{aligned}
$$

with

$$
F_{2}=\frac{\Lambda_{s}^{4}}{\Lambda_{s}^{4}+\left(q_{1}^{2}-m_{p}^{2}\right)^{2}}
$$

and
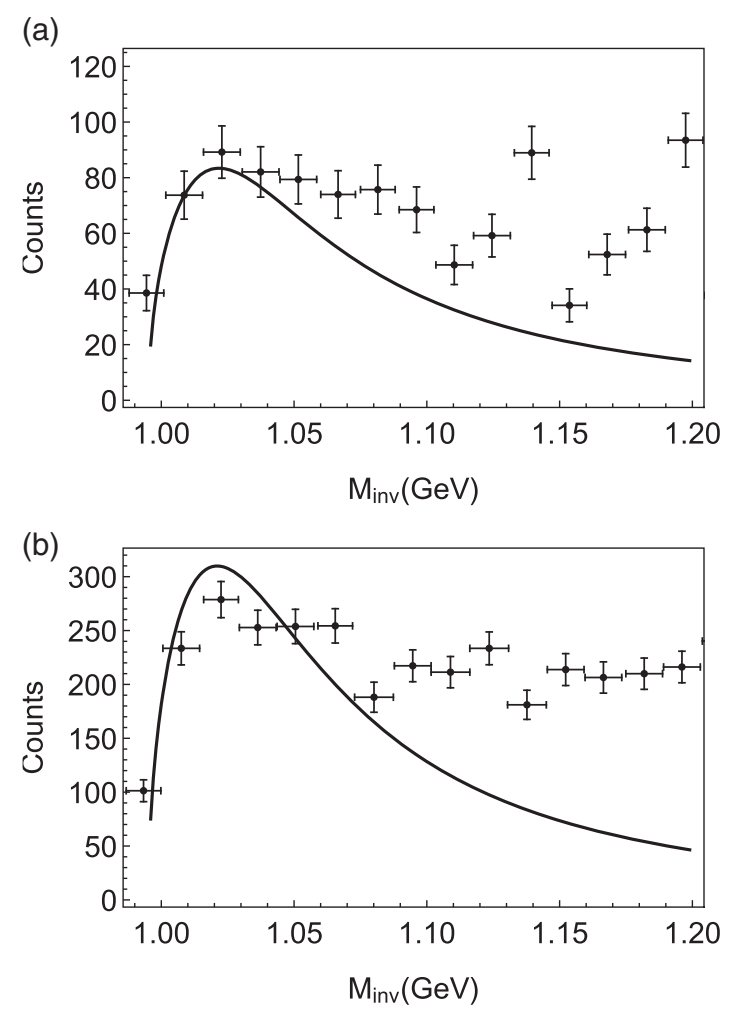

FIG. 6. Invariant mass distribution of $K^{0} \bar{K}^{0}$ of $\gamma p \rightarrow$ $p f_{0}(980) \rightarrow p K^{0} \bar{K}^{0}$ reaction for (a) $|t|<1.0 \mathrm{GeV}^{2}$ and (b) $|t|>1.0 \mathrm{GeV}^{2}$. The theoretical results are obtained with a contribution from the $s$-channel nucleon pole.

$$
\Gamma_{c}^{\mu}=-\frac{\not p_{1}}{p_{1} \cdot p_{2}} p_{2}^{\mu}
$$

which is obtained from a contact term and for keeping the scattering amplitude $\mathcal{M}_{s}$ gauge invariant [41,42].

The theoretical results of $K^{0} \bar{K}^{0}$ invariant mass distributions of the $\gamma p \rightarrow f_{0}(980) p \rightarrow K^{0} \bar{K}^{0} p$ reaction with the contribution from the $s$-channel nucleon pole are shown in Fig. 6, from which we see that we can explain the experimental measurements for both the $|t|<1 \mathrm{GeV}^{2}$ and $|t|>1 \mathrm{GeV}^{2}$ cases quite well, since there is no strong $t$ dependence factor $F_{1}$ in the $s$-channel process. The theoretical numerical results shown in Fig. 6 are obtained with $c_{1} g_{f_{0} p p}=7.5$ and $\Lambda_{s}=1.1 \mathrm{GeV}$.

One might think that the inclusion of higher nucleon excitations might improve the situation, since they have large mass and will give large contributions. However, at one certain photon energy $E_{\gamma}$, the propagator of the $s$-channel process is then just a constant. The estimation of the $K^{0} \bar{K}^{0}$ invariant mass distributions in our model is only sensitive to the production rate of the $f_{0}(980)$, and the nucleon pole term is sufficient for this purpose. By neglecting the contribution from other $N^{*}$ resonances, we can present a more general picture of the $s$-channel $f_{0}(980)$ production processes, though our results are more general than this would suggest. 


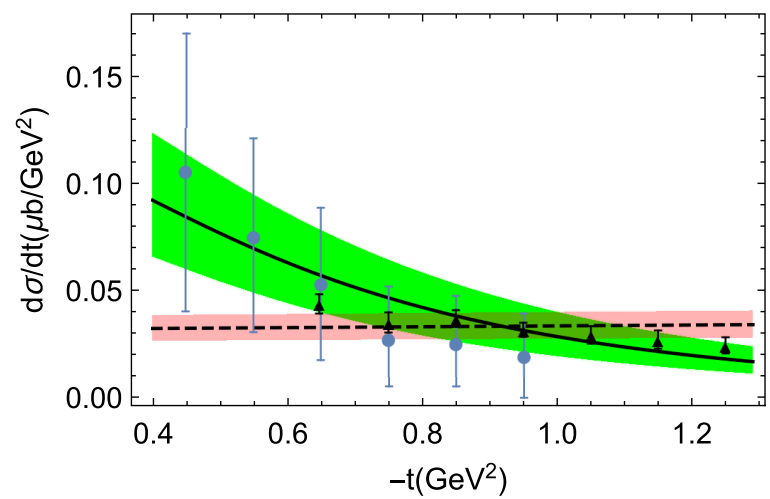

FIG. 7. Differential cross sections $d \sigma / d t$ of $\gamma p \rightarrow p f_{0}(980)$ reaction compared with the CLAS data from Ref. [15] (dot) and Ref. [17] (triangle).

On the other hand, we calculate the $d \sigma / d t$ for $\gamma p \rightarrow$ $p f_{0}(980)$ reaction with the above two different reaction mechanisms at the photon energy $E_{\gamma}=3.4 \mathrm{GeV}$. The numerical results are shown in Fig. 7, compared with the experimental data taken from Refs. [15,17]. The solid and dashed lines represent the result from the $t$ channel with $\Lambda_{c}=$ $1.07 \mathrm{GeV}$ and the $s$ channel with $g_{f_{0} p p}=4.3$, respectively. For the $t$-channel process, we consider the form factor effect on $d \sigma / d t$ with different cutoff parameter $\Lambda_{c}$. The green band in Fig. 7 is obtained with $\Lambda_{c}=1.07 \pm 0.03 \mathrm{GeV}$. From the figure, it can be clearly seen that the predicted $d \sigma / d t$ have a strong dependence on the $\Lambda_{c}$. For the $s$-channel process, since the predicted $d \sigma / d t$ depend only on $g_{f_{0} p p}$, we know very limited information about it. We show the predicted results obtained with $g_{f_{0} p p}=4.3 \pm 0.4$ in Fig. 7 with the pink band.

From Fig. 7, one can see that both the $t$-channel mechanism and the $s$-channel process can describe fairly well the current experimental data. However, the line shapes of these two different reaction mechanisms are sizably different. The slope of the results for the $s$-channel process is flatter than in the case of the $t$-channel $\rho^{0}$ and $\omega$ exchange. We hope that this feature may be used to determine the reaction mechanism of $\gamma p \rightarrow p f_{0}(980)$ reaction.

\section{SUMMARY}

In this work, we have studied the $\gamma p \rightarrow$ $p f_{0}\left[f_{0}(980), f_{0}(1500)\right] \rightarrow p K^{0} \bar{K}^{0}$ reactions near threshold within an effective Lagrangian approach. The $K^{0} \bar{K}^{0}$ invariant mass distributions are evaluated, where the two kaons have been separated in $S$ wave decaying from the scalar mesons $f_{0}(980)$ and $f_{0}(1500)$. It is shown that the $t$ channel $\rho^{0}$ and $\omega$ exchange processes can describe the experimental data on the $\gamma p \rightarrow p f_{0}(1500) \rightarrow p K^{0} \bar{K}^{0}$ reaction, while the $s$-channel process is favored for the $\gamma p \rightarrow$ $p f_{0}(980) \rightarrow p K^{0} \bar{K}^{0}$ reaction, since the $t$-channel mechanism for the $f_{0}(980)$ photoproduction fails to reproduce the experimental measurements. Furthermore, it is found that the theoretical numerical results for the $\gamma p \rightarrow p f_{0}(980)$ differential cross section, $d \sigma / d t$, of the two different reaction mechanisms are sizeably different. It is expected that the theoretical results can be tested by further experimental measurements at CLAS [26].

Finally, we would like to stress that, thanks to the important role played by the non- $t$-channel process in the $\gamma p \rightarrow p f_{0}(980)$ reaction, accurate data for this reaction can be used to improve our knowledge about the reaction mechanism of this reaction and also the nature of $f_{0}(980)$. This work constitutes a first step in this direction.

\section{ACKNOWLEDGMENTS}

This work is partly supported by the National Natural Science Foundation of China under Grants No. 11475227, No. 11522539, No.11675131, No. 11675091, No. 11735003 , and No. 11835015, the fundamental Research Funds for the Central Universities and the Youth Innovation Promotion Association CAS No. 2016367.
[1] E. Oset et al., Int. J. Mod. Phys. E 25, 1630001 (2016).

[2] F. K. Guo, C. Hanhart, U. G. Meiner, Q. Wang, Q. Zhao, and B. S. Zou, Rev. Mod. Phys. 90, 015004 (2018).

[3] J. D. Weinstein and N. Isgur, Phys. Rev. Lett. 48, 659 (1982).

[4] F. E. Close, N. Isgur, and S. Kumano, Nucl. Phys. B389, 513 (1993).

[5] J. A. Oller and E. Oset, Nucl. Phys. A620, 438 (1997); A652, 407(E) (1999).

[6] J. A. Oller and E. Oset, Phys. Rev. D 60, 074023 (1999).

[7] J. Nieves and E. Ruiz Arriola, Nucl. Phys. A679, 57 (2000).
[8] V. Baru, J. Haidenbauer, C. Hanhart, Y. Kalashnikova, and A. E. Kudryavtsev, Phys. Lett. B 586, 53 (2004).

[9] J. R. Pelaez, Phys. Rev. Lett. 92, 102001 (2004).

[10] F. Giacosa and G. Pagliara, Phys. Rev. C 76, 065204 (2007).

[11] H. J. Lee, N. I. Kochelev, and Y. Oh, Phys. Rev. D 87, 117901 (2013).

[12] M. Tanabashi et al. (Particle Data Group), Phys. Rev. D 98, 030001 (2018).

[13] M. Albaladejo and J. A. Oller, Phys. Rev. Lett. 101, 252002 (2008).

[14] V. Crede and C. A. Meyer, Prog. Part. Nucl. Phys. 63, 74 (2009). 
[15] M. Battaglieri et al. (CLAS Collaboration), Phys. Rev. Lett. 102, 102001 (2009).

[16] M. Battaglieri et al. (CLAS Collaboration), Phys. Rev. D 80, 072005 (2009).

[17] S. Lombardo et al. (CLAS Collaboration), Phys. Rev. D 98, 052009 (2018).

[18] D. C. Fries, P. Heine, H. Hirschmann, A. Markou, E. Seitz, H.-J. Behrend, W. P. Hesse, W. A. McNeely, and T. Miyachi, Nucl. Phys. B143, 408 (1978).

[19] D. P. Barber et al., Z. Phys. C 12, 1 (1982).

[20] C. R. Ji, R. Kaminski, L. Lesniak, A. Szczepaniak, and R. Williams, Phys. Rev. C 58, 1205 (1998).

[21] E. Marco, E. Oset, and H. Toki, Phys. Rev. C 60, 015202 (1999).

[22] M. L. L. da Silva and M. V. T. Machado, Phys. Rev. C 87, 065201 (2013).

[23] V. E. Tarasov, W. J. Briscoe, W. Gradl, A. E. Kudryavtsev, and I. I. Strakovsky, Phys. Rev. C 88, 035207 (2013).

[24] A. Donnachie and Y. S. Kalashnikova, Phys. Rev. C 93, 025203 (2016).

[25] M. L. L. da Silva and M. V. T. Machado, Phys. Rev. C 86, 015209 (2012).

[26] S. Chandavar et al. (CLAS Collaboration), Phys. Rev. C 97, 025203 (2018).

[27] H. Nagahiro, L. Roca, and E. Oset, Eur. Phys. J. A 36, 73 (2008).
[28] H. Nagahiro, L. Roca, E. Oset, and B. S. Zou, Phys. Rev. D 78, 014012 (2008).

[29] R. Machleidt, K. Holinde, and C. Elster, Phys. Rep. 149, 1 (1987).

[30] N. I. Kochelev, M. Battaglieri, and R. De Vita, Phys. Rev. C 80, 025201 (2009).

[31] J. J. Xie and E. Oset, Eur. Phys. J. A 51, 111 (2015).

[32] C. Cheng, J. J. Xie, and X. Cao, Commun. Theor. Phys. 66, 675 (2016).

[33] J. J. Xie, Phys. Rev. C 92, 065203 (2015).

[34] J. A. Oller, E. Oset, and J. R. Pelaez, Phys. Rev. D 59, 074001 (1999); 60, 099906(E) (1999); 75, 099903(E) (2007).

[35] J. A. Oller, Nucl. Phys. A727, 353 (2003).

[36] Q. Zhao, Phys. Lett. B 659, 221 (2008).

[37] Q. Zhao, B.S. Zou, and Z. B. Ma, Phys. Lett. B 631, 22 (2005).

[38] F. E. Close and Q. Zhao, Phys. Rev. D 71, 094022 (2005).

[39] Q. Wang, G. Li, and Q. Zhao, Int. J. Mod. Phys. A 27, 1250135 (2012).

[40] C. D. Lü, U. G. Meissner, W. Wang, and Q. Zhao, Eur. Phys. J. A 49, 58 (2013).

[41] J. J. Xie and J. Nieves, Phys. Rev. C 82, 045205 (2010).

[42] H. Haberzettl, K. Nakayama, and S. Krewald, Phys. Rev. C 74, 045202 (2006). 